\title{
Cardiovascular Health, Testosterone, and Oxandrolone: Leveraging the Myotrophic-Androgenic Ratio in Males with a Sarcopenic Obese Phenotype
}

\author{
Nashawi $\mathbf{M}^{1 *}$, Ahmed MS ${ }^{1}$, Ahmad $\mathrm{M}^{2}$, Issa $\mathrm{O}^{2}$ and \\ Abualfoul $\mathbf{M}^{3}$ \\ ${ }^{1}$ Department of Medicine-Cardiology, University of Texas \\ Health Science Center San Antonio, Terxas \\ ${ }^{2}$ Department of Biology, The University of Texas at \\ Arlington, Texas \\ ${ }^{3}$ Department of Internal Medicine, Methodist Dallas \\ Medical Center, Texas \\ *Corresponding author: Mouhamed Nashawi, \\ Department of Medicine-Cardiology, University of Texas \\ Health Science Center San Antonio, Division of Medicine- \\ Cardiology, MC 7872 UT Health San Antonio 7703 Floyd \\ Curl Dr., San Antonio, 78229-3900, Texas
}

Received: May 12, 2021; Accepted: J une 04, 2021; Published: J une 11, 2021

\begin{abstract}
Anabolic-Androgenic Steroids (AAS) are a group of organic compounds that include testosterone, or related compounds that induce similar effects by serving as structural analogues. Because of their propensity to induce gene expression that promotes protein synthesis, increased lean body mass, and strength, they have found utility in medicine to aide patients with chronic wasting syndromes, deficiencies in growth stature, and trauma recovery (e.g., burns). Contemporary off-label use of these classes of agents are also being used in anti-aging capacities under clinical supervision, and those with cardiovascular deficits related to metabolic derangement. Nevertheless, as hormones, testosterone and its analogues have systemic effects and their glut can be deleterious to global organs, namely the heart. Chronic utilization of these agents can be seen in domains of competitive physical activities given their performance enhancing effects. Associated with this abuse in particular have been ubiquitous clinical accounts of Major Adverse Cardiovascular Events (MACE), chronic hypertension, dyslipidemia, and left ventricular remodeling given the pleiotropic effects of testosterone and its analogues. One agent in particular, oxandrolone, a synthetic AAS, has an interesting profile as it has a biological disposition to more anabolic and metabolic effects compared to other AAS, with less profuse androgenic properties. There has been evidence to show that even oxandrolone supplementation may show promise in improving peripheral homeostasis conducive to positive cardiovascular health, especially in obese patients with features of metabolic syndrome, a condition related to endocrinological dysfunction and aberrant adiposity. In this commentary we will review the effects of this AAS with a commentary on cardiovascular physiology constructed around translational biology and clinical data. Commentaries such as the latter are scant in the literature and offer perspectives crucial to understanding the intersections between habitus, physiologic status, and the heart. Overall, oxandrolone shows promise related to its pharmacology in patients with low muscle tone and significant adiposity, namely cardiometabolic profiles if administered with clinical prudence due to its novel structure, metabolism, and effects.
\end{abstract}

Keywords: Oxandrolone; Cardiovascular; Heart; Testosterone; Estrogen; Aromatization

\section{Introduction}

Testosterone is a steroid derivative from cholesterol that serves multiple biological functions such as the induction of protein synthesis in myocytes, the mitigation of protein loss, promotion of bone density, and the development of secondary sexual characteristics in pubertal stages [1,2]. Part of the testosterone metabolism pathway includes conversion to its more potent counterpart Dihydrotesterone (DHT), by the enzyme $5 a$-reductase distributed throughout the body. As a non-polar compound, testosterone and DHT have the ability to cross the cellular membrane and binds to their intracellular receptor, the Androgen Receptor (AR) in the cytoplasm. Subsequent translocation to the nuclear membrane of affected cells sees these compounds primarily serve as transcription factors that bind to DNA promotor sequences, thus regulating gene expression to exert their pleiotropic effects (Figure 1). Research has also shown that testosterone and DHT binding to cellular AR can exert effects without direct genomic interaction through the regulation of cytoplasmic cell-signaling cascades and ion-flux, resulting in phosphorylation and activation of molecular constituents that ultimately yield a cellular effect congruent with the effects of testosterone, DHT, and related analogues of AAS [3-5].

\section{Testosterone and the Heart- Implications in Senescence and Sarcopenic Obesity}

Male status has been cited in ubiquitous reports as a risk for elevated cardiovascular disease risk [6]. It has been highly suggested that testosterone may be responsible for these effects as early studies have shown that Testosterone Replacement Therapy (TRT) has been associated with an increase risk in cerebrovascular events and MACE, 


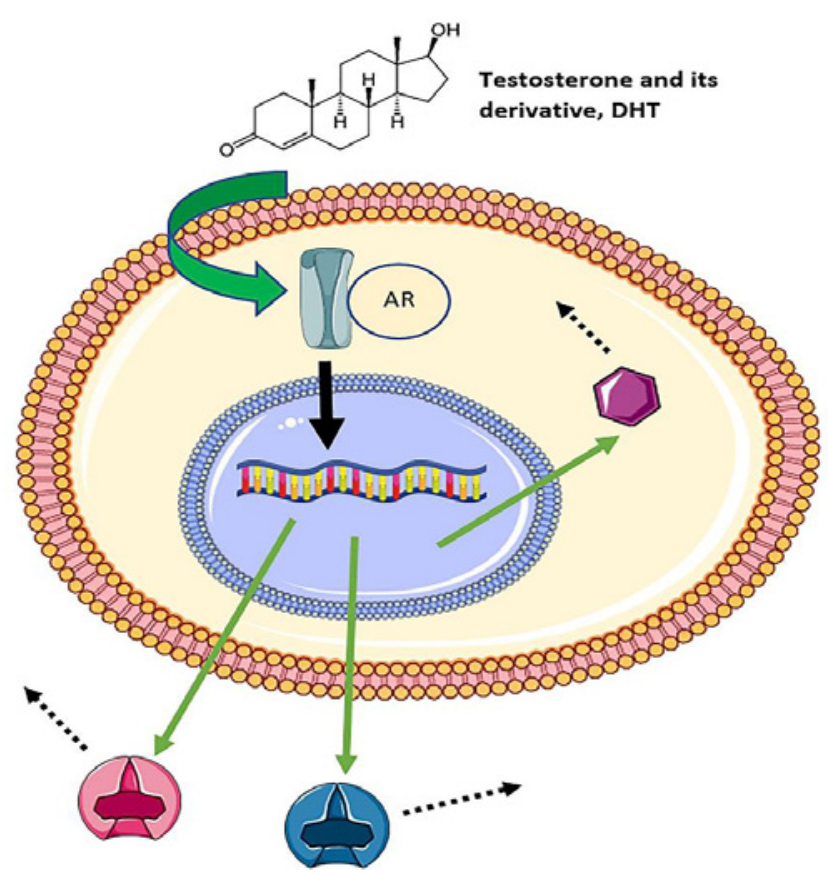

Figure 1: The Effects of Testosterone on Intracellular Androgen Receptor Binding and Genomic Modulation with Subsequent Physiological Effects. Adapted from Servier Medical Art by Servier, which is licensed under a Creative Commons Attribution 3.0 Unported License.

however subsequent studies have not validated this relationship with much credence [7]. In fact, it has been shown that testosterone may exert cardioprotective effects via pleiotropic effects. For example, Jankowska et. al, assessed 208 men with congestive heart failure versus 366 healthy control males and found that low testosterone was associated with all New York Heart Association (NYHA) classes of heart failure, with further studies showing that low testosterone confers poorer prognosis and increased mortality [8,9]. However, heart failure, has been increasingly recognized as a syndrome with peripheral implications, raising the notion whether these peripheral nodes related to heart failure have downstream effects leading to low testosterone, or whether low testosterone promotes a physiologic state related to failure of electromechanical synchrony and pumping related to heart failure [10]. One of the purported mechanisms is via stimulation of the sarcoendoplasmic $\mathrm{Ca}^{2+}$-ATPase, resulting in relaxation of the cardiomyocytes while increasing cardiac contraction $[9,11]$.

Testosterone has been traditionally implicated in the promotion of dyslipidemia via the reduction of High-Density Lipoprotein (HDL) and increase in Low-Density Lipoprotein (LDL), especially in men. The translational biology behind this principle is the notion that testosterone induces increased expression and viability of endogenous hepatic lipase, which is already naturally increased at baseline in men [12]. This enzyme is produced by the liver, and its presence has been found on the luminal surface of the sinusoidal endothelium [13]. Hepatic lipase promotes the phospholipid and triacylglycerol hydrolysis, affecting plasma lipoprotein flux [14]. The hydrolysis of these lipoproteins results in modulation of HDL-Cholesterol (HDL-C) subclass densities, which are amenable to uptake by the liver, resulting in a net decrease of serum HDL-C. HDL-C has been shown to have some antithrombotic and anti-inflammatory properties, with these effects having more weight in patients with attributes of metabolic syndrome, such as impaired glucose metabolism [15]. Moreover, in patients with atherosclerotic arteries, the endogenous metabolism of HDL-C has been believed to contribute to the egress of cholesterol from lipid-laden macrophages (pathologically known as "foam cells"), to the hepatic sinusoids for metabolism and secretion as bile constituents [16]. This process, known as "reverse cholesterol transport", is considered the hallmark cardioprotective effect of HDL-C in patients at risk for atherosclerosis (i.e, sarcopenic obese patients). The same propensity for hepatic lipase to hydrolyze HDL-C also manifests in Low-Density Lipoprotein Cholesterol (LDL-C). However, hydrolysis from a more buoyant, large family of LDL particles, dubbed "pattern A" to smaller, higher density particles, dubbed "Pattern B" is associated with increased cardiovascular disease as these lower density particles have an increased proclivity to penetrate the endothelium and undergo oxidation as oxidized LDL [17]. This starts an inflammatory cascade of atherogenesis and is considered a cardiovascular risk factor as this atherosclerotic plaque may rupture or promote subsequent thrombus formation, all resulting in the possibility of MACE if such structures are unstable and find themselves dislodged to vessels of significance to the myocardium or other end-organs (i.e, the brain, causing cerebrovascular accidents) [18]. Nevertheless, there is conflicting evidence for this mechanism, as it has been replicated in supraphysiologic testosterone environments, and there are mixed results of testosterone and lipid distribution in the hypogonadal population, such as those with sarcopenic obesity [19-24].

An increasing body of work has shown that testosterone may actually exert some cardioprotective functions. For example, one study by Svartberg et. al showed that lower levels of testosterone are actually inversely correlated to the magnitude of atherosclerosis in 
men with and without cardiovascular disease $(\mathrm{P}=0.0008)$ as measured by age-adjusted intimal medial thickness of the right carotid artery using high-resolution B-mode and color doppler/pulsed wave doppler ultrasonography [25]. Observations that give credence to the notion that normal testosterone function can promote a healthy lipid profile is observed in studies that show that the reductions of HDL-C seen with testosterone modulation to normal levels is diminutive relative to the more deleterious fractions of lipids that are deleterious to endothelial function and are pro-atherosclerotic, such as the dense LDL-C molecular subtypes [26]. Evidence giving support for this can be seen in one study that used flow-mediated vasodilation of the brachial artery via ultrasonography in 178 consecutive male patients (mean age \pm SD: $47 \pm 15$ years), showing significant correlation with total and free testosterone levels $(\mathrm{p}<0.001)$, also suggesting that very low testosterone may have deleterious effects on endothelial function [27]. Another study by Malkin et. al assessing 900 men found that both total and bioavailable testosterone is clinically significant in those with Coronary Artery Disease (CAD) and those without, with a prevalence of hypogonadotropic function of $24 \%$ in the CAD group [28].

Lower testosterone has been associated with decreased skeletal muscle mass in men, as is observed clinically in untrained elderly men. However, evidence has shown that testosterone stimulates anabolic factors related to skeletal muscle mass such as mTOR (a serine/threonine protein kinase that promotes protein synthesis, with auxiliary roles as a tyrosine kinase receptor site for insulin receptor activation for the trafficking of amino acids to promote a positive nitrogen balance conducive to the anabolic state) and Akt (a serine/threonine protein kinase which promotes inactivation of apoptotic proteins, preserving anabolism), which are both implicated in signal transduction pathways related to myocyte proliferation and survival [29]. Decreased levels of skeletal muscle mass overall would represent a reduced capacity for insulin-mediated glucose disposal via decreased global GLUT4, resulting in improper glucose metabolism. Moreover, testosterone has been shown to increase GLUT4 expression independent of baseline skeletal muscle mass implying a dual effect of glucose disposal in testosterone [30,31]. Evidence for this phenomenon can be appreciated by Srikanthan et al. who showed that graded levels of increase skeletal muscle mass to total body weight was inversely proportional to homeostatic models of insulin resistance [32]. This is of importance to the sarcopenic patient presenting with obesity as decreased skeletal muscle mass related to low testosterone may be linked to impaired glucose metabolism. A glut of glucose has been shown to induce the formation of Reactive Oxygen Species (ROS) through stimulation of the mitochondrial electron transport chain, disrupting the redox capacity of the mitochondria via NADPH dysregulation, generating deleterious molecular constituents. Moreover, nonenzymatic glycation products that result from interactions with glucose and amine groups, also activates ROS production. The result in the endothelium, is decreased Nitric Oxide (NO) though attenuation of eNOS (endothelial Nitric Oxide Synthase), leading to vasoconstriction, higher blood pressure, increased afterload, and increased cardiovascular work leading to potential Left Ventricular Hypertrophy (LVH) and subsequently, MACE [33,34]. Moreover, increased systemic ROS stemming from increased glucose as a consequence of decreased skeletal muscle mass promotes increased adhesion molecule expression such as
Intracellular Adhesion Molecule-1 (ICAM-1) and Vascular Adhesion Molecule-1 (VCAM-1), resulting in increased inflammatory cell recruitment, lipid deposition, and smooth muscle proliferation [35]. The latter are all detrimental sequelae in atherosclerosis and increase the likelihood of a potential plaque rupture and MACE in a patient. Studies that have assessed skeletal muscle mass using urinary creatinine excretion as a surrogate have shown that increased urinary creatinine in a graded fashion was associated with reductions in cardiovascular disease and mortality [36]. Furthermore, clinical analyses have also bolstered the notion the skeletal muscle mass is a clinically significant and relevant variable in the evaluation of different types of heart disease [37-42]. While testosterone has the propensity to affect the heart peripherally via deleterious metabolic and cellular mechanisms a la skeletal muscle mass diminution, there are also intracardiac ramifications to low testosterone of relevant to the role of the heart as an electromechanical pump. For example, decreased skeletal mass from testosterone results in diminished exercise capacity. For a given supply of oxygen, decreased metabolic activity from lack of skeletal muscle mass results in decreased oxygen extraction, which is known as the Arteriovenous Oxygen Difference $\left(\mathrm{AVO}_{2}\right)$ [43]. This variable represents the difference between arterial and venous compartments within the body, and is directly correlated to peak $\mathrm{VO}_{2}$ (the observed zenith of the rate of oxygen consumption under parameters of physical exercise) via the formula:

$$
\text { Peak } \mathrm{VO}_{2}=\mathrm{HR} * \mathrm{SV}^{*} \mathrm{AVO}_{2}
$$

Where HR represents the heart rate, and SV represents the cardiac stroke volume (the differential of end diastolic volume and end systolic volume within a cardiac cycle measured as the volume of blood ejected during ventricular contraction per cardiac cycle). Peak $\mathrm{VO}_{2}$ is especially vital as a variable to gauge cardiovascular mortality and morbidity, with clinical evidence showing its utility in assessing recurrent admission rates of cardiovascular etiology, all-cause mortality in heart failure, and the demand for heart transplantation [44-48].

Corroborating this is evidence from a study by Nichols et al. assessed male patients with coronary heart disease (CHD) through cardiopulmonary testing to achieve peak $\mathrm{VO}_{2}$ [49]. Baseline skeletal muscle mass was demonstrated through Dual X-ray Absorptiometry Assessment (DEXA), and was defined in this study as appendicular lean mass (the mass of skeletal muscle in both arms and legs, reported as skeletal muscle index or SMI). Moreover, SMI was indexed to total body mass and reported as a variable defined as proportion of appendicular skeletal muscle mass to total body mass (ASM\%). Using cutoffs of SMI $<7.26 \mathrm{~kg} / \mathrm{m}^{2}$, or ASM\% $<25.72 \%$ to demarcate low skeletal muscle mass, SMI and ASM\% were observed to have a positive correlation as marked by Pearson correlation coefficient $(\mathrm{r}=0.431$ and $\mathrm{r}=0.473$ respectively, $\mathrm{P}<0.001$ for both variables assessed). Moreover, ASM\% exhibited an inverse relationship with 5-year all-cause mortality risk $(r=-0.365 ; \mathrm{P}=0.006)$, indicating that not only absolute skeletal muscle mass is associated with poor quality of life.

A clinical study by Cicoira et al. used similar cardiopulmonary exercise tests and preliminary DEXA assessments of lean muscle mass in patients with Chronic Heart Failure (CHF) [50]. Heart failure stratification by NYHA classification was done via echodoppler studies to assess cardiac chamber dynamics and anatomy. 
Univariate analysis that NYHA class was significantly correlated with peak $\mathrm{VO}_{2}(\mathrm{P}<0.0001)$. Skeletal muscle mass and peak $\mathrm{VO}_{2}$ showed similar correlation $(\mathrm{r}=0.70, \mathrm{P}<0.0001)$. The importance of this revelation has shown that skeletal muscle mass has correlations with cardiopulmonary fitness and cardiovascular disease burden across multiple cardiovascular pathologies, highlighting the scope that low testosterone may negatively impact poor heart health with skeletal muscle mass showing a role as a surrogate variable.

\section{The Sarcopenic Obesity Phenotype and Testosterone}

Our commentary on the systemic effects of androgenic constituents such as testosterone thus far have served the purpose of illustrating the effects of these steroid hormones on the systemic physiology, namely that of which is relevant to healthy cardiovascular function. Moreover, the latter was laid to open a dialogue on how these processes are relevant to the patient with sarcopenia and significant obesity. Sarcopenic obesity is a clinical condition characterized by a habitus composed of low skeletal muscle mass tone and bulk or myocyte function in the context of increased adiposity, namely central adiposity in the majority of these cases [51]. Concomitant with this phenotype are components of metabolic syndrome, such as Type 2 Diabetes Mellitus (T2DM), dyslipidemia (high serum triglycerides and low HDL-C), hypertension, and increased central abdominal obesity [52-54]. One study by Lu et al. showed that sarcopenic obesity confers a high risk for metabolic syndrome (Odds Ratio [OR] 11.59 [95\% confidence interval [CI] 6.72-19.98]) [54]. Moreover, there are theories that the promotion of adiposity in the context of low skeletal muscle mass has a component of endocrinological senescence [55]. These factors overlay many of the components of low testosterone in males as discussed previously [56]. Such sequelae can be observed in Figure 2 for a global reference to the dialogue of testosterone, physiologic derangements, and eventually, our dialogue on how oxandrolone has the propensity to impact these metabolic nodes.

While serving a role as a reservoir of energy in the form of lipids, adipose tissue with a central predisposition within habitus has been known to induce systemic inflammation and has received increasing attention as a particular domain within the endocrine system [5759]. For example, adipose tissue is metabolically active and has the propensity to secrete an array of intercellular signaling peptides and proteins, commonly referred to as adipokines- or cytokines of adipose origin. The obesity in sarcopenia has been observed to promote a status of inflammation that results in dysregulation of normal adipocyte regulation, resulting in ectopic lipid deposition in myocytes causing lipotoxicity via impairment of mitochondrial $\beta$-oxidation, insulin regulation, and the promotion of ROS, exacerbating the release of deleterious myokines that further decreases skeletal muscle mass and the concomitant cardiovascular impairments associated with them [60]. In healthy conditions, adipose tissue secretes adiponectin, an adipokine responsible for the stimulation of $5^{\prime}$-AMP-Activated Protein Kinase (AMPK) leading to endothelial synthase (eNOS) function [61]. Moreover, adiponectin has been shown to promote hydrogen sulfide $\mathrm{H}_{2} \mathrm{~S}$ production, a known modulator of voltagegated potassium channels [62]. Leptin is another adipokine that upregulates the production of pro-inflammatory cytokines, such as TNF- $\alpha$ [63]. The induction of TNF- $\alpha$ from sarcopenic obesity inhibits adiponectin while negatively impacting myocyte mitochondrial biogenesis and myogenesis, causing a vicious cycle within sarcopenia obesity of decreased muscle mass and increased adiposity [64]. Overexpression of TNF- $a$ from sarcopenic obesity also downregulates eNOS, further exacerbating hemodynamics, while promoting a negative ionotropic cardiac state $[65,66]$. Moreover, there is evidence to show that it promotes pathologic vascular and valvular calcification $[67,68]$. There is preliminary evidence that markedly elevated levels of TNF- $\alpha$ may inhibit testosterone via direction action on the pituitary gland, leading to transient losses in the cardio protective effects of normal serum testosterone levels [69]. Lastly, TNF- $\alpha$ in excess has been shown to promote central obesity, the most negative allocation of adiposity in the context of negative adipokine secretion, further amplifying these negative sequelae[70]. In sarcopenic obesity, these processes are pathologically disturbed as there is a component of senescence and dysregulation to adipose tissue, with ramifications of the downstream players in adipokines such as adiponectin being implicated in vascular tone, vasomotor function, and responsiveness to ischemia via eNOS, or through antiarrhythmic effects a la $\mathrm{H}_{2} \mathrm{~S}$, just to name a few examples [71-75]. It is apparent that the sarcopenic obese phenotype, coupled with its ability to hamper exercise and the pathways it induces, promotes a phenotype conducive not only to ageing and metabolic syndrome, but many avenues of MACE via pro-atherosclerotic, increased arrhythmic potential, ventricular remodeling, coronary artery disease, and vascular deterioration to name a few [76-78].

As we have seen thus far, the body composition of sarcopenic obesity is both conducive to a status of low testosterone as well as deleterious cardiovascular health. Commentaries within the literature give recommendations for testosterone replacement therapy under clinical supervision to improve the body composition that results in inflammation and metabolic derangements leading to such cardiovascular demise [79]. Current clinical recommendations for the treatment of sarcopenic obesity advocate for lifestyle changes, such as diets that provide adequate protein for the flux of amino acids resulting in skeletal muscle mass maintenance, in addition to nutritional options that promote a low glycemic index profile as to mitigate hyperglycemia as the duality of these nutrient states attempt to oppose the habitus of sarcopenic obesity [80]. Coupled with the latter is the recommendation for the initiation of exercise training programs, namely resistance training to promote insulin sensitivity and anabolic status amenable to skeletal muscle hypertrophy in the setting of a positive nitrogen balance via diet- which has the propensity to increase to promote lipolysis and combatting the sarcopenic obese phenotype- interestingly, irrespective of baseline basal metabolic rate [81-83].

While lifestyle interventions have shown promise in improving the sarcopenic phenotype or mitigating its exacerbation overtime, there represents a proclivity for pharmacotherapeutic augmentation to optimize results. Credence for this opportunity in clinical practice has been validated as testosterone replacement therapy with lifestyle changes has been shown to superiority over lifestyle changes or TRT alone [84]. Repletion of testosterone may therefore provide an avenue towards mitigation of heart disease as a result of sarcopenia. Moreover, sarcopenic obesity may reach severity in some instances, that respiratory function is compromised, making exercise capacity difficult to achieve until attenuation of the sarcopenic obese 

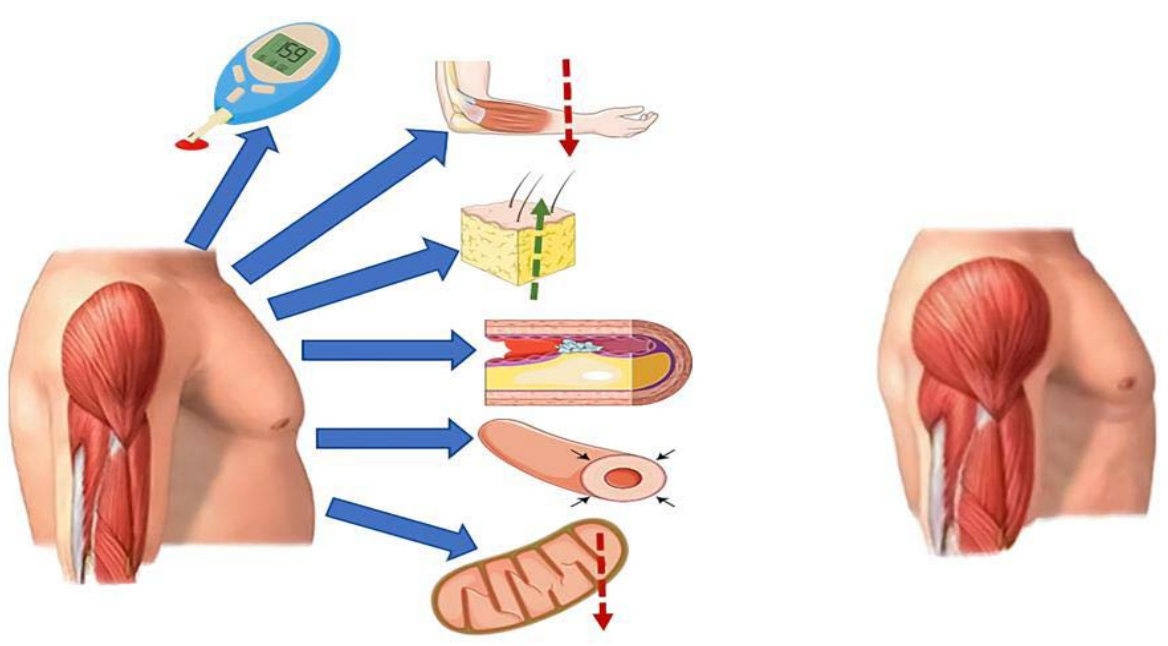

Figure 2: The Cardiometabolic Sequelae of Sarcopenic Obesity (Low Skeletal Muscle Mass, Increased Adiposity- Left, Relative to Fit Phenotype on Right). Adapted from Servier Medical Art by Servier, which is Licensed under a Creative Commons Attribution 3.0 Unported License and Public Domain Access.

phenotype makes maximization of lifestyle interventions possible $[85,86]$. This phenomenon is believed to be due to atypical protein turnover and a metabolic resistance to anabolism resulting in exercise intolerance, further warranting an inquiry on the significance of potential hormonal restoration to make such lifestyle changes viable [87].

One double-blind placebo-controlled crossover study by Kapoor et al. recruited men with features of sarcopenia, namely men with insulin resistance and hypogonadism [88]. In this study, a biweekly intramuscular testosterone dose of $200 \mathrm{mg}$ versus placebo for 3 months in random order was administered, succeeded by a washout period of 1 month before an alternation of treatment phase. It was shown that relative to placebo testosterone therapy improved glycated hemoglobin, HbA1c $(-0.37 \pm 0.17 \%, \mathrm{P}=0.03)$, waist circumference $(-1.63 \pm 0.71 \mathrm{~cm}, \mathrm{P}=0.03)$, waist/hip ratio $(-0.03 \pm 0.01, \mathrm{P}=0.01)$, and total cholesterol $(-0.4 \pm 0.17 \mathrm{mmol}, \mathrm{P}=0.03)$, highlighting the propensity for testosterone to not improve anthropomophric variables related to sarcopenia as well as the associated metabolic defects of interest to heart health. Unfortunately, there are scant studies with human subjects with sarcopenic obesity and testosterone, despite the redundancy of clinical commentaries imploring their prospect as a therapeutic option [89].

One potential pitfall in the use of direct testosterone for in patients with sarcopenic obesity is the notion that the bioavailability of testosterone may be in jeopardy in patients with significant adiposity. For example, one interventional study assessing lipoaspirate explants of subcutaneous adipose tissue in a small cohort of obese versus normal patients showed a significant increase in intracellular testosterone in obese individuals in the setting of simultaneous androgen receptor deficiency, implying sequestration of free testosterone in obese individuals [90]. This sequestration of testosterone in dysfunctional adiposity implies that testosterone may not achieve maximal physiological effects in the sarcopenic obese patient, and other avenues of body composition improvement must be pursued before the sarcopenic obese patient is receptive to testosterone therapy to improve their habitus and in essence, cardiovascular disease burden. A second potential impediment to direct testosterone supplementation in this group of patients is that non-sequestered testosterone may not circulate as testosterone at all in patients with a significant mass of adipose tissue. That is because in addition to their adiponectin secreting roles, adipose tissue (with central adiposity in particular) expresses significant levels of aromatase, an enzyme that promotes the conversion of testosterone to $17 \beta$-estradiol, which does not exhibit the same cardioprotective or anabolic profile of testosterone in men with sarcopenic obesity, further decreasing the level of bioavailable testosterone in the sarcopenic obese phenotype [70]. A study by $\mathrm{Xu}$ et. al studying the association between testosterone conversion and obesity in individuals with significant abdominal obesity (defined as a waist-to-hip circumference ratio $>0.9$ ) [91]. Serum aromatase were measured by ELISA and showed increased levels in individuals with significant central obesity (mean $4.86 \mathrm{ng} / \mathrm{ml}$, average Standard Deviation (SD) of 1.56) relative to individuals without significant central adiposity (mean $3.64 \mathrm{ng} / \mathrm{ml}$, average standard deviation of 1.44. $\mathrm{P}<0.0001$ ). Concordance with aromatase to testosterone and estradiol ratios to give context to the physiological ramifications of hormone modulation in those with pathologic habitus was also pursued with testosterone and Estradiol (E2) measured via blood sample with subsequent immunoassay. It was shown that nonobese individuals had a mean T/E2 of 0.231 with an SD of 0.117 units. Meanwhile obese patients had a meant/E2 ratio of 0.147 and an SD of 0.68 , highlighting not only was absolute testosterone was lower in patients with marked central obesity, but the distribution attributed to physiological differences as marked by the SD was small, implying the power of aromatase to dampen serum testosterone levels to a significant degree. While TRT in the sarcopenic obese has physiological impediments to regulate gene expression and enact pleiotropic effects to its maximal potential due to physiological impediments, alternatives to the aromatization dilemma with the prospect of a novel pleiotropic profile due to its unique biochemical structure, we will discuss the utility of oxandrolone as an alternative AAS therapy in the sarcopenic obese to showcase its ability to mimic testosterone in its cardioproective ability. 

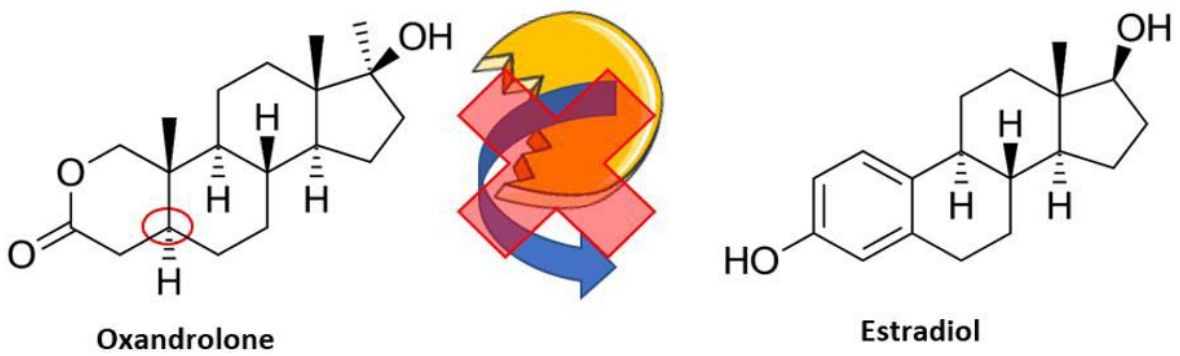

Figure 3: Pre-Reduction of Oxandrolone at the $5 \alpha$ (Red Circle) Precludes Aromatase Enactment for Conversion to Estradiol by Inhibiting Aromatic Bond Formation and 19-Methyl Group Oxidation. Adapted from Servier Medical Art by Servier, which is Licensed under a Creative Commons Attribution 3.0 Unported License and Public Domain Access.

\section{Oxandirolone, Sarcopenic Obesity, and Cardiovascular Health}

Oxandrolone is an AAS that has been traditionally used in the promotion of anabolism in settings such as burn trauma or growth stature defects. Due to its similar structure to testosterone, it has an affinity for the androgen receptor and has similar androgenic effects, albeit weaker relative to exogenous testosterone. Oxandrolone has been observed to have roughly a 10:1 ratio of myotrophic:androgenic ratio compared to testosterone and its ester, methyltestosterone, implying its utility in metabolic modulation without concern for excess vigilance for side effects of overt virilization during its application, of which does not offer much aide in improving habitus nor metabolic parameters in sarcopenia [92,93]. Moreover, due to its structure having already undergone reduction at the $5 \alpha$ position, it is not a substrate for aromatase, nullifying the yield of estradiol from its usage (Figure 3).

As can also be seen in Figure 3, the 19 methyl-group of oxandrolone is also retained as compared to the analogous cyclic group of estradiol, further giving credence that oxandrolone intrinsically does not promote conversion to estrogen derivatives a la aromatase activity, even in the settings of profound obesity. An immediate benefit to the mitigation of estradiol in the sarcopenic obese is that estradiol has been associated with fluid retention $[94,95]$. This has been validated by studies observing the effects of estradiol therapy on subjects exposed to differential saline concentrations, with audits of free water clearance, osmole compartments, and plasma aldosterone concentration [94]. Although no analogue to this study has been observed in sarcopenic men, there is a mutual element of senescence in this study and the sarcopenic obese male. Unregulated fluid retention in those severe endocrinological impairments may suffer from exacerbations of concomitant heart disease associated with sarcopenic obesity and low testosterone, such as the cases of heart failure discussed earlier due to increased peripheral resistance from volume overload $[96,97]$. From a clinical perspective, aromatized TRT leading to potential estradiol conversion and fluid retention may also impact stratification of sarcopenic obesity as studies using different imaging modalities for evaluation of body composition implicate edema as a erroneously non-adipose mass, which may lead to differential assessments of true sarcopenic obesity magnitude by healthcare providers $[98,99]$. The latter may impact treatment guidelines, graded nutritional counseling, and exercise plans based on an altered perception of phenotype relative to physiological status.
Oxandrolone exerts further cardioprotective effects compatible to the sarcopenic obese phenotype via the promotion of hepatic ketogenesis [100]. As previously stated, oxandrolone has the propensity to increase hepatic lipase, and by proxy, nonesterified fatty acids. These constituents would promote the production of ketones in patients with insulin impairment, as the trafficking of glucose to the intracellular compartment as a nutrient is mitigated, in line with the "starvation in the midst of plenty" moniker of insulin resistance and T2DM (both highly associated with the sarcopenic obese phenotype). Related to the notion of sarcopenic obesity, subcutaneous fat decreases with age and increases in the visceral abdominal fat compartment, which has been associated with dyslipidemia [101]. Further studies by Vega et al. noted that in endogenous hypertriglyceridemia (for example, in sarcopenic obesity), resulted in impaired hepatic ketogenesis in men with significant abdominal obesity [102]. Theories include that the induced hypertriglyceridemia may occur due to non-esterified fatty acids entering hepatic sinusoids may undergo re-esterification detracting from the ketogenic pathways, observed activation of hepatic lipogenesis and decreased hepatic lipase, or defective oxidative from pathologic dyslipidemia. One study using oxandrolone supplementation noted a decrease of triglycerides of $50 \mathrm{mg} / 100 \mathrm{~mL}$ in roughly half of the patients without significant side effects seven months after treatment cessation indicating that dyslipidemia associated with sarcopenic obesity may be a target for Oxandrolone [103]. Oxandrolone has the propensity to correct this dyslipidemia and promote ketogenic flux through its effects of hepatic lipase. With cardiovascular implications, increased ketones may exert cardioprotective properties through the "thrifty substrate hypothesis" as first commented in SGLT2 inhibitor study [104]. It is believed that the ketones promoted by oxandrolone utilization, such as $\beta$-hydroxybutyrate are amenable to uptake to the myocardium, and oxidized with an affinity to fatty acids. This substrate has been shown to improve oxygen flux consumption and mitochondrial efficiency, mitigation of ROS, and improved cardiometabolic outcomes which may further give credence to oxandrolone as a cardioprotective agent similar to testosterone. Cardiovascular ketone body oxidation is metabolically favorable to the heart, as it has a favorable ATP-toOxygen ratio relative to other metabolic substrates [105]. Moreover, ketone body metabolism has been observed to impact membrane excitability in animal models by modulating voltage-gated potassium channels, in direct opposition to adiponectin in the sarcopenic obese phenotype as mentioned earlier [105]. Testosterone exhibits effects similar to these via modulation of ion channels affecting 
the electromechanical pump of the heart [106-108]. Nevertheless, oxandrolones propensity to similarly exhibit antiarrhythmic effects akin to testosterone and it should be noted that these effects run parallel with novel attributes such as the promotion of lipolysis through ketogenesis and its propensity to become impervious to aromatization to estradiol, unlike testosterone.

Oxandrolone affects sarcopenic obesity through another node, the muscular component of this habitus derangement through anabolic and protective effects. The $\beta$-hydroxybutyrate stimulated from hepatic lipase activation by oxandrolone has also been shown to have anticatabolic effects on skeletal muscle through the modulation of anti-apoptotic pathways [109]. Moreover, there is an element of myocyte preservation via the maintenance of positive nitrogen balance (a surrogate for amino acid density) [110]. One study assessing the ability for oxandrolone to fuel protein synthesis was observed in a study assessing its supplementation, which has showed increased fractional synthetic rate of protein turnover by $44 \%$ in one cohort $(\mathrm{P}<0.05)$, with no changes in amino acid efflux from myocyte based on isotopic data and muscle data [111]. Moverover, polymerase chain reaction has shown increased myocyte ribosomal messenger RNA, indicating increased gene transcription of the androgen receptor [111]. Further data It has also been shown to improve exercise capacity in patients with mitochondrial dysfunction seen in sarcopenic obesity and senescence as supported by studies showing ketone flux in subjects indexed to maximal oxygen consumption and graded improvements in maximal walking speed, distance, and time to exhaustion, allowing the sarcopenic obese patient to train and move to a hypertrophic phenotype with respect to skeletal muscle [112]. This evidence shows that ketones as a result of oxandrolone supplementation has effects beyond improvements in body composition and adipose tissue reduction through improvements in myocyte function (affecting both culprits in sarcopenic obesity). Oxandrolone, just like testosterone, can be used to increase the peak $\mathrm{VO}_{2}$ and through increased skeletal muscle mass, and as a source of glucose uptake, mitigating heart disease on multiple domains. The implications of these metabolic changes hold a purpose in augmenting the first-line lifestyle changes such as exercise which improves heart health. Thus far, oxandrolone has been shown to work as an AAS to exhibit cardioprotective effects via arrhythmic equilibrium, improved metabolic work, reduction of potential edema potential, and creating an environment conducive to decreased inflammatory cardiotoxicity in addition to exercise.

Another aspect of positive cardiovascular health outcome possibly related to the anabolic properties of oxandrolone is ischemic heart disease. Anemia has been associated with sarcopenic obesity as revealed by a cross-sectional study assessing Hemoglobin (Hgb) in patients with and without sarcopenic obesity with a prevalence of $39 \%$ in this specific cohort, with decreased levels compared to controls $(\mathrm{P}=0.001)$ [113]. Cardiovascular ramifications associated with anemia include ischemic heart disease as a result of a decreased overall oxygen delivery inclination [114]. Credence for this phenomenon is given through the findings of the Myocardial Ischemia National Audit Project (MINAP), a registry that collects data on adults admitted to hospital affiliates in aspects of the United Kingdom with a presentation of Acute Coronary Syndrome (ACS). A retrospective cohort study of 422,855 patients revealed a prevalence of anemia in $27.7 \%$ of patients with ACS, and that in patients with
ACS, a diagnosis of anemia exhibits independent association with 30day (OR 1.28, 95\% CI 1.22-1.35) and 1-year mortality (OR 1.31, 95\% CI 1.27-1.35). Such results given alarm as this suggests a link between sarcopenic obesity and ischemic heart disease [115]. Theories for this phenomenon in sarcopenia include muscular atrophy resulting in hampered iron metabolism, decreased skeletal muscle mass leading to decreased myokines that are associated with red marrow attenuation, of lipotoxicity from adipose glut findings its way to bone marrow in a pathologic state [116-119]. Nevertheless, oxandrolone has the propensity to stimulate RBC synthesis via androgen receptor increases (which is achieved through mRNA increase as a result of DNA transcription modulation as shown in Figure 1 and mentioned previously), and the androgen receptor has been shown to bind to targets that promote erythropoietin stimulation, resulting in increased red blood cells through oxandrolone [120]. This increased red blood cell count not only results in mitigation of myocardial ischemia, but also further reinforce the first-line treatment of sarcopenic obesity that includes exercise as a modality of habitus restructuring, and an increased red blood cell count increases global oxygen carrying capacity resulting in increased exercise tolerance. This yet again shows an advantage that oxandrolone can mimic testosterone, without the worry of aromatization in the obese patient to estradiol. While estradiol also has been shown to aide in myocardial ischemia, its role is more in ischemic-reperfusion applications and there has been some speculation that as an estrogen group, there is an increased increase of venous thromboembolism which may lead to ACS itself, another reason why to mitigate estradiol if possible alternatives impervious to aromatization exist $[121,122]$.

\section{Conclusion: Oxandrolone Side Effects and Looking Forward}

While the main advantage discussed thus far regarding oxandrolone revolve around its ability to exhibit cardioprotective effects similar to testosterone, it does not exhibit aromatization to estradiol, maximizing yield in the sarcopenic obese patient. Moreover, there are more cardiometabolic properties such as attenuation of the inflammasome, peripheral protection via endocrinological modulation such as ketogenesis, glucose uptake via skeletal muscle mass, and hepatic lipase effects. Moreover, similar to testosterone, auxiliary metabolic effects occur such as the resolution of the anemic problem in the sarcopenic obese, and impacting nitrogen balance resulting in oxidative protection of the mitochondria and decreased global inflammation of relevance to the heart. These have given evidence for oxandrolone in mitigation of cardiopulmonary decline, heart failure, myocardial ischemia, antiarrhythmic effects, and attenuation of atherosclerosis via low-dosage supplementation equalizing the ratios of serum lipids. Nevertheless, side effects of all AAS classes including oxandrolone include left ventricular hypertrophy and cardiac remodeling from overt usage in the form of increased afterload, hypertension, and cardiac work if used in excess and not supervised with prudence in the sarcopenic obese. Because testosterone is the gold standard regarding its studied effects, but undergoes aromatization to estradiol, which antagonizes the effects of the primary male sex androgens, research into oxandrolone has a therapeutic option to allow sarcopenic obesity to mitigate adiposity resulting in amenability to conventional testosterone therapy should be studied in patients. These may unlock therapeutic strategies that 
gives stepwise approaches to complex metabolic problems with cardiac implications, as these solutions mirror the complex state of sarcopenic obesity.

\section{References}

1. Lam T, Poljak A, McLean M, Bahl N, Ho KK, Birzniece V. Testosterone prevents protein loss via the hepatic urea cycle in human. European journal of endocrinology. 2017; 176: 489-496.

2. Kraemer WJ, Ratamess NA, Nindl BC. Recovery responses of testosterone growth hormone, and IGF-1 after resistance exercise. Journal of Applied Physiology 2017; 122: 549-558.

3. Heinlein CA, Chang C. The roles of androgen receptors and androgen binding proteins in nongenomic androgen actions. Molecular endocrinology 2002; 16: 2181-2187.

4. Fix C, Jordan C, Cano P, Walker WH. Testosterone activates mitogenactivated protein kinase and the cAMP response element binding protein transcription factor in Sertoli cells. Proceedings of the National Academy of Sciences 2004; 101: 10919-10924.

5. Cheng J, Watkins SC, Walker WH. Testosterone activates mitogen-activated protein kinase via Src kinase and the epidermal growth factor receptor in sertoli cells. Endocrinology 2007; 148: 2066-2074.

6. Tsang S, Liu J, Ming Wong T. Testosterone and cardioprotection against myocardial ischemia. Cardiovascular \& Haematological Disorders-Drug Targets (Formerly Current Drug Targets-Cardiovascular \& Hematological Disorders) 2007; 7: 119-125

7. Halpern JA, Brannigan RE. Testosterone deficiency. Jama. 2019; 322 : 1116-1116.

8. Jankowska EA, Biel B, Majda J, et al. CLINICAL PERSPECTIVE. Circulation 2006; 114: 1829-1837

9. Wehr E, Pilz S, Boehm BO, März W, Grammer T, Obermayer-Pietsch B. Low free testosterone is associated with heart failure mortality in older men referred for coronary angiography. European journal of heart failure 2011 13: $482-488$.

10. Jahng JWS, Song E, Sweeney G. Crosstalk between the heart and peripheral organs in heart failure. Experimental \& Molecular Medicine. 2016 48: e217-e217.

11. Tsang S, Wong SSC, Wu S, Kravtsov GM, Wong T-M. Testosteroneaugmented contractile responses to $\alpha 1$-and $\beta 1$-adrenoceptor stimulation are associated with increased activities of RyR, SERCA, and NCX in the heart. American Journal of Physiology-Cell Physiology. 2009; 296: C766-C782.

12. Auwerx JH, Marzetta CA, Hokanson JE, Brunzell JD. Large buoyant LDL like particles in hepatic lipase deficiency. Arteriosclerosis: An Official Journal of the American Heart Association, Inc 1989; 9: 319-325.

13. Soppert J, Lehrke M, Marx N, Jankowski J, Noels H. Lipoproteins and lipids in cardiovascular disease: from mechanistic insights to therapeutic targeting Advanced Drug Delivery Reviews. 2020.

14. Han H, Dai D, Wang W, et al. Impact of serum levels of lipoprotein lipase hepatic lipase, and endothelial lipase on the progression of coronary artery disease. Journal of Interventional Medicine. 2019; 2: 16-20.

15. Ebara S, Marumo M, Mukai J, Ohki M, Uchida K, Wakabayashi I. Relationships of oxidized HDL with blood coagulation and fibrinolysis in patients with type 2 diabetes mellitus. Journal of thrombosis and thrombolysis 2018; 45: 200 205.

16. Ouimet M, Barrett TJ, Fisher EA. HDL and reverse cholesterol transport: Basic mechanisms and their roles in vascular health and disease. Circulation research 2019; 124: 1505-1518.

17. Pokharel $Y$, Tang $Y$, Bhardwaj B, et al. Association of low-density lipoprotein pattern with mortality after myocardial infarction: Insights from the TRIUMPH study. Journal of clinical lipidology. 2017; 11: 1458-1470.

18. Ivanova EA, Myasoedova VA, Melnichenko AA, Grechko AV, Orekhov AN. Small dense low-density lipoprotein as biomarker for atherosclerotic diseases. Oxidative medicine and cellular longevity. 2017; 2017.

19. Singh $A B$, Hsia $S$, Alaupovic $P$, et al. The effects of varying doses of $T$ on insulin sensitivity, plasma lipids, apolipoproteins, and C-reactive protein in healthy young men. The Journal of Clinical Endocrinology \& Metabolism. 2002; 87: 136-143.

20. Vermeulen A. Low levels of sex hormone-binding globulin and testosterone are associated with smaller, denser low density lipoproteins in normoglycemic men-Commentary. Journal of Clinical Endocrinology and Metabolism 1998; 83: $1822-1823$.

21. Tan KCB, Shiu SWM, Kung AWC. Alterations in hepatic lipase and lipoprotein subfractions with transdermal testosterone replacement therapy. Clinical endocrinology. 1999; 51: 765-769.

22. Saad F, Haider A, Haider KS, Doros G, Traish AM. Effect of testosterone therapy on cardiovascular risk factors, major adverse cardiovascular events and mortality in men with functional hypogonadism and cardiovascular disease in a real-world registry. European Heart Journal. 2020; 41: ehaa9463221.

23. Yassin A, Haider A, Haider KS, et al. Testosterone therapy in men with hypogonadism prevents progression from prediabetes to type 2 diabetes: eight-year data from a registry study. Diabetes Care 2019; 42: 1104-1111.

24. Khripun I, Vorobyev S, Khripun A, Kogan M, Demidova A, Belousov I. Effect of testosterone on lipid metabolism and endothelium in men with type 2 diabetes mellitus and hypogonadism. European Heart Journal. 2020; 41: ehaa946-3827.

25. Svartberg J, Von Mühlen D, Mathiesen E, Joakimsen O, Bønaa $\mathrm{KH}$ Stensland-Bugge E. Low testosterone levels are associated with carotid atherosclerosis in men. Journal of internal medicine. 2006; 259: 576-582.

26. Morris PD, Channer KS. Testosterone and cardiovascular disease in men. Asian journal of andrology. 2012; 14: 428-435.

27. Akishita M, Hashimoto $M$, Ohike $Y$, et al. Low testosterone level is an independent determinant of endothelial dysfunction in men. Hypertension research. 2007; 30: 1029-1034.

28. Malkin CJ, Pugh PJ, Morris PD, Asif S, Jones TH, Channer KS. Low serum testosterone and increased mortality in men with coronary heart disease. Heart. 2010; 96: 1821-1825.

29. White JP, Gao S, Puppa MJ, Sato S, Welle SL, Carson JA. Testosterone regulation of $\mathrm{Akt} / \mathrm{mTORC1/FoxO3a}$ signaling in skeletal muscle. Molecular and cellular endocrinology. 2013; 365: 174-186.

30. Mitsuhashi K, Senmaru T, Fukuda T, et al. Testosterone stimulates glucose uptake and GLUT4 translocation through LKB1/AMPK signaling in 3T3-L1 adipocytes. Endocrine. 2016; 51: 174-184.

31. Fink J, Matsumoto M, Tamura Y. Potential application of testosterone replacement therapy as treatment for obesity and type 2 diabetes in men Steroids. 2018; 138: 161-166.

32. Srikanthan P, Karlamangla AS. Relative muscle mass is inversely associated with insulin resistance and prediabetes. Findings from the third National Health and Nutrition Examination Survey. The Journal of Clinical Endocrinology \& Metabolism. 2011; 96: 2898-2903.

33. MacCarthy PA, Grieve DJ, Li J-M, Dunster C, Kelly FJ, Shah AM. Impaired endothelial regulation of ventricular relaxation in cardiac hypertrophy: role of reactive oxygen species and NADPH oxidase. Circulation 2001; 104: $2967-$ 2974.

34. Moris D, Spartalis M, Spartalis E, et al. The role of reactive oxygen species in the pathophysiology of cardiovascular diseases and the clinical significance of myocardial redox. Annals of translational medicine. 2017; 5.

35. Kaneto $\mathrm{H}$, Katakami N, Matsuhisa M, Matsuoka T-A. Role of reactive oxygen species in the progression of type 2 diabetes and atherosclerosis. Mediators of inflammation. 2010; 2010.

36. Oterdoom LH, Gansevoort RT, Schouten JP, de Jong PE, Gans ROB Bakker SJL. Urinary creatinine excretion, an indirect measure of muscle mass, is an independent predictor of cardiovascular disease and mortality in 
the general population. Atherosclerosis. 2009; 207: 534-540.

37. Lopez PD, Nepal P, Akinlonu A, et al. Low skeletal muscle mass independently predicts mortality in patients with chronic heart failure after an acute hospitalization. Cardiology. 2019; 142: 28-36.

38. Kang DO, Park SY, Choi BG, et al. Prognostic impact of low skeletal muscle mass on major adverse cardiovascular events in coronary artery disease: a propensity score-matched analysis of a single center all-comer cohort. Journal of clinical medicine. 2019; 8: 712.

39. Paternostro G, Camici PG, Lammerstma AA, et al. Cardiac and skeletal muscle insulin resistance in patients with coronary heart disease. A study with positron emission tomography. The Journal of clinical investigation. 1996; 98: 2094-2099.

40. Lavie CJ, Forman DE, Arena R. Bulking up skeletal muscle to improve hear failure prognosis. American College of Cardiology Foundation Washington, DC. 2016

41. Tyrovolas S, Panagiotakos D, Georgousopoulou E, et al. Skeletal muscle mass in relation to 10 year cardiovascular disease incidence among middle aged and older adults: the ATTICA study. J Epidemiol Community Health. 2020; 74: 26-31.

42. Srikanthan $\mathrm{P}$, Horwich TB, Tseng $\mathrm{CH}$. Relation of muscle mass and fat mass to cardiovascular disease mortality. The American journal of cardiology 2016; 117: 1355-1360.

43. Lambert CP. Is It Time for a "Muscle-Centric" View of Maximal Oxygen Consumption during Exercise? Journal of Exercise Physiology Online. 2019; 22.

44. Mancini DM, Eisen H, Kussmaul W, Mull R, Edmunds Jr LH, Wilson JR Value of peak exercise oxygen consumption for optimal timing of cardiac transplantation in ambulatory patients with heart failure. Circulation. 1991; 83: $778-786$.

45. Palau P, Domínguez E, Núñez E, et al. Peak exercise oxygen uptake predicts recurrent admissions in heart failure with preserved ejection fraction. Revista Española de Cardiología (English Edition). 2018; 71: 250-256.

46. Shafiq A, Brawner CA, Aldred HA, et al. Prognostic value of cardiopulmonary exercise testing in heart failure with preserved ejection fraction. The Henry Ford HosplTal CardioPulmonary EXercise Testing (FIT-CPX) project American heart journal. 2016; 174: 167-172.

47. Chiaranda G, Myers J, Arena R, et al. Improved percent-predicted peak $\mathrm{VO}_{2}$ is associated with lower risk of hospitalization in patients with coronary heart disease. Analysis from the FRIEND registry. International journal of cardiology 2020; 310: 138-144.

48. Ahmad A, Corban M, Toya T, et al. Coronary Microvascular Function is Correlated With Peak Exercise Capacity in Patients With Unexplained Cardiac Exertion Symptoms and Non-obstructive Coronary Artery Disease. Circulation. 2020; 142: A14283-A14283.

49. Nichols S, O'Doherty AF, Taylor C, Clark AL, Carroll S, Ingle L. Low skeletal muscle mass is associated with low aerobic capacity and increased mortality risk in patients with coronary heart disease-a CARE CR study. Clinical physiology and functional imaging. 2019; 39: 93-102.

50. Cicoira M, Zanolla L, Franceschini L, et al. Skeletal muscle mass independently predicts peak oxygen consumption and ventilatory response during exercise in noncachectic patients with chronic heart failure. Journal of the American College of Cardiology. 2001; 37: 2080-2085.

51. Dos Santos L, Cyrino ES, Antunes M, Santos DA, Sardinha LB. Sarcopenia and physical independence in older adults: the independent and synergic role of muscle mass and muscle function. Journal of cachexia, sarcopenia and muscle. 2017; 8: 245-250.

52. Roh E, Choi KM. Health consequences of sarcopenic obesity: A narrative review. Frontiers in Endocrinology. 2020; 11: 332.

53. Polyzos SA, Margioris AN. Sarcopenic obesity. Hormones. 2018; 17: 321331.

54. Lu C-W, Yang K-C, Chang H-H, Lee L-T, Chen C-Y, Huang K-C. Sarcopenic obesity is closely associated with metabolic syndrome. Obesity research \& clinical practice. 2013; 7: e301-e307.

55. El Bizri I, Batsis JA. Linking epidemiology and molecular mechanisms in sarcopenic obesity in populations. Proceedings of the Nutrition Society. 2020.

56. Fui MNT, Dupuis $P$, Grossmann M. Lowered testosterone in male obesity: mechanisms, morbidity and management. Asian journal of andrology. 2014 16: 223.

57. Kahn CR, Wang G, Lee KY. Altered adipose tissue and adipocyte function in the pathogenesis of metabolic syndrome. The Journal of clinical investigation. 2019; 129: 3990-4000.

58. Russell AC, Kepka A, Trbojević-Akmačić I, et al. Increased central adiposity is associated with pro-inflammatory immunoglobulin $\mathrm{G} \mathrm{N}$-glycans. Immunobiology. 2019; 224: 110-115.

59. Ahluwalia A, Misto A, Vozzi F, et al. Systemic and vascular inflammation in an in-vitro model of central obesity. PLoS One. 2018; 13: e0192824.

60. Kalinkovich A, Livshits G. Sarcopenic obesity or obese sarcopenia: a cross talk between age-associated adipose tissue and skeletal muscle inflammation as a main mechanism of the pathogenesis. Ageing research reviews 2017; 35: 200-221.

61. Ghantous CM, Farhat R, Djouhri L, et al. Molecular mechanisms of adiponectin-induced attenuation of mechanical stretch-mediated vascular remodeling. Oxidative medicine and cellular longevity. 2020; 2020.

62. Shimizu Y, Polavarapu R, Eskla K-L, et al. Hydrogen sulfide regulates cardiac mitochondrial biogenesis via the activation of AMPK. Journal of molecular and cellular cardiology. 2018; 116: 29-40.

63. Soni AK, Pithadia AB, Deshpande SS, Suhagia BN. Endocrinological role of leptin in obesity and asthma. Population. 2019; 12: 14.

64. Yeap BB, Paul Chubb SA, Lopez D, Ho KKY, Hankey GJ, Flicker L. Associations of insulin-like growth factor-I and its binding proteins and testosterone with frailty in older men. Clinical endocrinology. 2013; 78: 752 759.

65. Yokoyama T, Vaca L, Rossen RD, Durante W, Hazarika P, Mann DL. Cellular basis for the negative inotropic effects of tumor necrosis factor-alpha in the adult mammalian heart. The Journal of clinical investigation 1993; 92: 2303-2312.

66. Nashawi M, Sheikh O, Battisha A, Mir M, Chilton R. Beyond the myocardium? SGLT2 inhibitors target peripheral components of reduced oxygen flux in the diabetic patient with heart failure with preserved ejection fraction. Heart Failure Reviews. 2020: 1-16.

67. Gonzalez Rodriguez A, Schroeder ME, Grim JC, et al. Tumor necrosis factor- $\alpha$ promotes and exacerbates calcification in heart valve myofibroblast populations. The FASEB Journal. 2021; 35: e21382.

68. Aghagolzadeh $\mathrm{P}$, Bachtler M, Bijarnia R, et al. Calcification of vascular smooth muscle cells is induced by secondary calciprotein particles and enhanced by tumor necrosis factor- $\alpha$. Atherosclerosis. 2016; 251: 404-414.

69. Mohamad N-V, Wong SK, Hasan WNW, et al. The relationship between circulating testosterone and inflammatory cytokines in men. The Aging Male. 2018.

70. Hong S-H, Choi KM. Sarcopenic obesity, insulin resistance, and their implications in cardiovascular and metabolic consequences. International journal of molecular sciences. 2020; 21: 494.

71. Swan KW, Song BM, Chen AL, et al. Analysis of decreases in systemic arterial pressure and heart rate in response to the hydrogen sulfide donor sodium sulfide. American Journal of Physiology-Heart and Circulatory Physiology. 2017; 313: H732-H743.

72. Zhang $\mathrm{L}$, Wang $\mathrm{Y}$, $\mathrm{Li} \mathrm{Y}$, et al. Hydrogen sulfide $\left(\mathrm{H}_{2} \mathrm{~S}\right)$-releasing compounds: therapeutic potential in cardiovascular diseases. Frontiers in pharmacology. 2018; 9: 1066.

73. Hong F-f, Liang X-y, Liu W, et al. Roles of eNOS in atherosclerosis treatment. Inflammation Research. 2019. 
74. Barbosa AM, Silva KSF, Lagares $\mathrm{MH}$, et al. Atherosclerosis: analysis of the eNOS (T786C) gene polymorphism. Genetics and Molecular Research. $2017 ; 16$.

75. Pabon MA, Manocha K, Cheung JW, Lo JC. Linking arrhythmias and adipocytes: insights, mechanisms, and future directions. Frontiers in physiology. 2018; 9: 1752.

76. Di lorio A, Di Blasio A, Napolitano G, Ripari P, Paganelli R, Cipollone F. High fat mass, low muscle mass, and arterial stiffness in a population of freeliving healthy subjects: The "al passo con la tua salute" project. Medicine. 2019; 98.

77. Livshits G, Kalinkovich A. Inflammaging as a common ground for the development and maintenance of sarcopenia, obesity, cardiomyopathy and dysbiosis. Ageing research reviews. 2019; 56: 100980.

78. Yang X, Woo J, Lui LT, et al. Cardiac Manifestations of Sarcopenia. The journal of nutrition, health \& aging. 2020; 24: 478-484.

79. Bouchonville MF, Villareal DT. Sarcopenic Obesity-How Do We Treat It? Current opinion in endocrinology, diabetes, and obesity. 2013; 20: 412.

80. Nabuco HCG, Tomeleri CM, Fernandes RR, et al. Effect of whey protein supplementation combined with resistance training on body composition muscular strength, functional capacity, and plasma-metabolism biomarkers in older women with sarcopenic obesity: A randomized, double-blind placebo-controlled trial. Clinical nutrition ESPEN. 2019; 32: 88-95.

81. Trouwborst I, Verreijen A, Memelink R, et al. Exercise and nutrition strategies to counteract sarcopenic obesity. Nutrients. 2018; 10: 605

82. Burd NA, McKenna CF, Salvador AF, Paulussen KJM, Moore DR. Dietary protein quantity, quality, and exercise are key to healthy living: a musclecentric perspective across the lifespan. Frontiers in nutrition. 2019; 6: 83.

83. Miller T, Mull S, Aragon AA, Krieger J, Schoenfeld BJ. Resistance training combined with diet decreases body fat while preserving lean mass independent of resting metabolic rate: A randomized trial. International journal of sport nutrition and exercise metabolism. 2018; 28: 46-54.

84. Barnouin Y, Armamento-Villareal R, Celli A, et al. Testosterone Replacement Therapy Added to Intensive Lifestyle Intervention in Older Men With Obesity and Hypogonadism. The Journal of Clinical Endocrinology \& Metabolism. 2021; 106: e1096-e1110.

85. Petermann-Rocha F, Yang S, Gray SR, Pell JP, Celis-Morales C, Ho FK Sarcopenic obesity and its association with respiratory disease incidence and mortality. Clinical Nutrition. 2020; 39: 3461-3466.

86. Sheean PM, Peterson SJ, Gomez Perez S, et al. The prevalence of sarcopenia in patients with respiratory failure classified as normally nourished using computed tomography and subjective global assessment. Journal of Parenteral and Enteral Nutrition. 2014; 38: 873-879.

87. Nilsson MI, Dobson JP, Greene NP, et al. Abnormal protein turnover and anabolic resistance to exercise in sarcopenic obesity. The FASEB Journal. 2013; 27: 3905-3916.

88. Kapoor D, Goodwin E, Channer KS, Jones TH. Testosterone replacement therapy improves insulin resistance, glycaemic control, visceral adiposity and hypercholesterolaemia in hypogonadal men with type 2 diabetes. European Journal of Endocrinology. 2006; 154: 899-906.

89. Scott D, Hirani V. Sarcopenic obesity. European Geriatric Medicine. 2016; 7: 214-219.

90. Di Nisio A, Sabovic I, De Toni L, et al. Testosterone is sequestered in dysfunctional adipose tissue, modifying androgen-responsive genes. International Journal of Obesity. 2020; 44: 1617-1625.

91. Xu X, Wang L, Luo D, et al. Effect of testosterone synthesis and conversion on serum testosterone levels in obese men. Hormone and Metabolic Research. 2018; 50: 661-670.

92. Kuhn CM. Anabolic steroids. Recent progress in hormone research. 2002; 57: 411-434.

93. Kiracofe B, Coffey R, Jones LM, et al. Incidence of oxandrolone induced hepatic transaminitis in patients with burn injury. Burns. 2019; 45: 891-897.
94. Stachenfeld NS, Dipietro L, Palter SF, Nadel ER. Estrogen influences osmotic secretion of AVP and body water balance in postmenopausal women. American Journal of Physiology-Regulatory, Integrative and Comparative Physiology. 1998; 274: R187-R195.

95. Yarrow JF, McCoy SC, Borst SE. Tissue selectivity and potential clinical applications of trenbolone (17ß-hydroxyestra-4, 9, 11-trien-3-one): a potent anabolic steroid with reduced androgenic and estrogenic activity. Steroids. 2010; 75: 377-389

96. Carbone S, Billingsley HE, Rodriguez-Miguelez $\mathrm{P}$, et al. Lean mass abnormalities in heart failure: the role of sarcopenia, sarcopenic obesity, and cachexia. Current problems in cardiology. 2019; 45: 100417.

97. Narumi T, Watanabe T, Kadowaki S, et al. Sarcopenia evaluated by fat-free mass index is an important prognostic factor in patients with chronic heart failure. European journal of internal medicine. 2015; 26: 118-122.

98. Deniz O, Cruz-Jentoft A, Sengul Aycicek G, et al. Role of ultrasonography in estimating muscle mass in sarcopenic obesity. Journal of Parenteral and Enteral Nutrition. 2020; 44: 1398-1406.

99. Silva AO, Karnikowski MGO, Funghetto SS, et al. Association of body composition with sarcopenic obesity in elderly women. International journal of general medicine. 2013; 6: 25.

100. Vega GL, Clarenbach JJ, Dunn F, Grundy SM. Oxandrolone enhances hepatic ketogenesis in adult men. Journal of Investigative Medicine. 2008; 56: 920-924.

101. Kim TN, Choi KM. The implications of sarcopenia and sarcopenic obesity on cardiometabolic disease. Journal of cellular biochemistry. 2015; 116: 1171 1178.

102. Vega GL, Dunn FL, Grundy SM. Impaired hepatic ketogenesis in moderately obese men with hypertriglyceridemia. Journal of investigative medicine. 2009; 57: 590-594.

103. Doyle AE, Pinkus NB, Green J. The use of oxandrolone in hyperlipidaemia Medical Journal of Australia. 1974; 1: 127-129.

104. Ferrannini E, Mark M, Mayoux E. CV protection in the EMPA-REG OUTCOME trial: a "thrifty substrate" hypothesis. Diabetes care. 2016; 39: 1108-1114.

105. Cotter DG, Schugar RC, Crawford PA. Ketone body metabolism and cardiovascular disease. American Journal of Physiology-Heart and Circulatory Physiology. 2013.

106. Seyrek M, Yildiz O, Ulusoy HB, Yildirim V. Testosterone relaxes isolated human radial artery by potassium channel opening action. Journal of pharmacological sciences. 2007; 103: 309-316.

107. Masuda K, Takanari H, Morishima M, et al. Testosterone-mediated upregulation of delayed rectifier potassium channel in cardiomyocytes causes abbreviation of QT intervals in rats. The Journal of Physiological Sciences. 2018; 68: 759-767.

108. Deenadayalu VP, White RE, Stallone JN, Gao X, Garcia AJ. Testosterone relaxes coronary arteries by opening the large-conductance, calciumactivated potassium channel. American Journal of Physiology-Heart and Circulatory Physiology. 2001; 281: H1720-H1727.

109. Koutnik AP, D'Agostino DP, Egan B. Anticatabolic effects of ketone bodies in skeletal muscle. Trends in Endocrinology \& Metabolism. 2019; 30: 227-229.

110. Thompson JR, Wu G. The effect of ketone bodies on nitrogen metabolism in skeletal muscle. Comparative Biochemistry and Physiology Part B: Comparative Biochemistry. 1991; 100: 209-216.

111. Sheffield-Moore M, Urban RJ, Wolf SE, et al. Short-term oxandrolone administration stimulates net muscle protein synthesis in young men. The Journal of Clinical Endocrinology \& Metabolism. 1999; 84: 2705-2711.

112. Monsalves-Alvarez M, Morales PE, Castro-Sepulveda $M$, et al. $\beta$-Hydroxybutyrate Increases Exercise Capacity Associated with Changes in Mitochondrial Function in Skeletal Muscle. Nutrients. 2020; 12: 1930.

113. Bani Hassan E, Vogrin S, Hernandez Viña I, Boersma D, Suriyaarachchi $P$, Duque G. Hemoglobin levels are low in sarcopenic and osteosarcopenic 
older persons. Calcified Tissue International. 2020; 107: 135-142.

114. Zeidman A, Fradin Z, Blecher A, Oster HS, Avrahami Y, Mittelman M Anemia as a risk factor for ischemic heart disease. Imaj-Ramat Gan. 2004 ;6: 16-18.

115. Kim J-H, Cho JJ, Park YS. Relationship between sarcopenic obesity and cardiovascular disease risk as estimated by the Framingham risk score. Journal of Korean medical science. 2015; 30: 264

116. Spargo E, Pratt OE, Daniel PM. Metabolic functions of skeletal muscles of man, mammals, birds and fishes: a review. Journal of the Royal Society of Medicine. 1979; 72: 921-925.

117. Hamrick MW. A role for myokines in muscle-bone interactions. Exercise and sport sciences reviews 2011; 39: 43

118. Schnyder S, Handschin C. Skeletal muscle as an endocrine organ: PGC-1 1 myokines and exercise. Bone. 2015; 80: 115-125.
119. Guo W, Pirtskhalava T, Tchkonia T, et al. Aging results in paradoxical susceptibility of fat cell progenitors to lipotoxicity. American Journal of Physiology-Endocrinology and Metabolism. 2007; 292: E1041-E1051.

120. McManus JF, Nguyen NYN, Davey RA, et al. Androgens stimulate erythropoiesis through the DNA-binding activity of the androgen receptor in non-hematopoietic cells. European journal of haematology. 2020; 105: 247-254.

121. Hale SL, Birnbaum Y, Kloner RA. $\beta$-estradiol, but not $\alpha$-estradiol, reduces myocardial necrosis in rabbits after ischemia and reperfusion. American heart journal. 1996; 132: 258-262.

122. Yeap BB. Rates of conversion of testosterone to estradiol may influence risk of venous thromboembolism in men. The Journal of Clinical Endocrinology \& Metabolism. 2021. 